\title{
INSTRUCTIONS TO AUTHORS
}

These instructions follow the latest edition of the Publication Manual of the American Psychological Association (http://www.apastyle.org/). Authors of research manuscripts are strongly encouraged to follow relevant reporting guidelines as outlined in the special editorial: Use of Reporting Guidelines in Scientific Writing: PRISMA, CONSORT, STROBE, STARD and Other Resources, Brain Impairment, 12, 1-21 (journals.cambridge.org/bim/reporting_guidelines). A statement confirming ethics approval should be included in all research manuscripts.

\section{Manuscript Submissions}

Cambridge Journals Online: http://journals.cambridge. org/action/displayJournal?jid=BIM

All articles are refereed. Papers submitted to the journal must not have been published previously or submitted for publication to any other journal and must represent original work.

\section{Manuscript Preparation}

Manuscripts must be presented double spaced in a clear, readable typeface (Times preferred), in an A4-size document with $3 \mathrm{~cm}$ margins. Number all pages except the figures, beginning with the first page. Your submission should have a separate title page bearing the name(s) and affiliation(s) of the contributing author(s), and an abstract of no more than 200 words with keywords typed on a separate page. An e-mail address and/or fax/telephone numbers are required for contact purposes and should be stated following the corresponding author's address in a footnote on the title page.

\section{Headings}

Provide headings that subdivide the paper into its key areas. Reports of empirical studies will generally follow a sequence of headings, including method, results and discussion. Review, theoretical, case study and other papers need not follow such a format but should provide a logical structure and appropriate section headings.

\section{Style}

The written paper should be logical, economical and precise in structure and use of language.

\section{Tables}

Reserve tables for important data directly related to the content of the paper. A well-constructed table should enable data to be isolated from the text and presented in a way that enables the reader to quickly see patterns and relationships of the data not readily discernible in the text. Use brief but explanatory table titles. The table title is placed at the top of the table. Include each table on a separate sheet. When constructing tables use tabs to space your columns as this will make it much easier to typeset the table in the text.

\section{Figures}

Figures should be prepared to the correct size (max. width up to $120 \mathrm{~mm}$ ) and each one supplied as an individual file, separate to the manuscript Word file. Include placement instructions in the Word document, such as 'Insert Figure 1 here'. The figure title is placed at the bottom of the figure. Prior to sending artwork, the separate files of figures, graphs, illustrations, should be printed by the author to test that the fonts have been embedded correctly and there is no distortion in the artwork as any such faults cannot be corrected by the publisher.

\section{Referencing}

References and citations should follow the APA format. Some examples to assist you are provided below.

\section{Citations in text}

For a single author: In a recent review, Smith (1992) suggested that ... A recent review (Smith, 1992) suggested that ... In 1992, Smith suggested that ... For two authors: In a recent review, Smith and Watson (1992) suggested that ... A recent review (Smith \& Watson, 1992) suggested that ... In 1992, Smith and Watson suggested that ... When a work has three, four, or five authors: Cite all authors the first time the reference occurs; thereafter, the name of the first author followed by et al. (e.g., Smith et al., 1991). The full list of authors must be cited in the list of references at the end of the paper. If use of the 'et al.' format gives rise to confusion, with another work of the same year and with the same first author, the references should be differentiated by the use of alphabet sequence following the publication year (e.g., Smith et al., 1991a; Smith et al., 1991b). When a work has six or more authors: Cite only the surname of the first author, followed by et al.; in the reference list, provide initials and surnames of the first six authors followed by an elipsis and the final author. General: Within a paragraph the year need not be repeated in subsequent citations of the same study provided the study cannot be confused with other studies cited in the paper. When citing several studies within the same set of parentheses, the following format should be adhered to '... several studies (Brooks, 1974a, 1974b; Cairns et al., 1992; Miller, in press; Smith, 1992; Tarter et al., 1985, 1987; Watson \& Smith, 1990) have reported that ...'

\section{Reference List}

Chapter in an edited book: Heilman, K.M., Watson, R.T., \& Valenstein, E. (1985). Neglect and related disorders. In K.M. Heilman \& E. Valenstein (Eds.), Clinical neuropsychology (2nd ed., pp. 243-293), New York: Oxford University Press. Complete book: Lezak, M.D. (1983). Neuropsychological assessment (2nd ed.). New York: Oxford University Press. Paper published in a journal: Grober, E., Gitlin, H.L., Bang, S., \& Buschke, H. (1992). Implicit and explicit memory in young, old and demented adults. Journal of Clinical and Experimental Neuropsychology, 14, 298-316. Published psychological or other test: Kertesz, A. (1982). Western Aphasia Battery. New York: Grune \& Stratton. Unpublished paper presented at a conference: Walsh, K.W. (1986, August). Bridging the gaps in clinical neuropsychology: The applied scientist model. Paper presented at the 21st Annual Conference of the Australian Psychological Society, James Cook University, Townsville, Queensland. Theses: Author, (Year). Title. Type of thesis, Institution, Location of Institution. General: Papers in the Reference List should be listed alphabetically by first author, and then by date. Single author entries precede multiple author entries beginning with the same surname. References with the same first author and different second or third authors are arranged alphabetically by the surname of the second author, and so on. 


\section{VOLUME 13}

\section{NUMBER 3}

\section{DECEMBER 2012 \\ CONTENTS}

\section{Articles}

Responsiveness of Instruments to Assess Disorders of

Consciousness:A Literature Review

Emmah J. Doig and Amanda T. Lane-Brown

Healthcare Consumers' Need for Brain-injury Services:

The Critical Importance of Timing in Planning Future Services

Anna O'Callaghan, Lindy McAllister, and Linda Wilson

Pitfalls of Psychogenic Non-epileptic Status Epilepticus

Hulya Karatas, F. Irsel Tezer, Nese Dericioglu, and Serap Saygi

Comparing Performance of Young Adults on a

Computer-based Version of the Austin Maze and

the Conventional Form of the Test

Adam McKay, Shuzi Lee, Rene Stolwyk, and Jennie Ponsford

Bringing Single-case Methodology into the Clinic to Enhance

Evidence-based Practices

Robyn L. Tate, Vanessa Aird, and Christine Taylor

\section{Controlled Trials Forum}

Acceptance and Commitment Therapy (ACT) for

Psychological Adjustment after Traumatic Brain Injury:

Reporting the Protocol for a Randomised Controlled Trial

Diane L. Whiting, Grahame K. Simpson, Hamish J. McLeod,

Frank P. Deane, and Joseph Ciarrochi 